\title{
Co-Occurring Post Traumatic Stress Disorder and Opioid Dependence: A Role for Buprenorphine
}

\author{
Itai Danovitch ${ }^{*}, 1,2$, Sandra Comer $^{2}$ and Maria Sullivan ${ }^{2}$ \\ ${ }^{I}$ Department of Psychiatry, Cedars-Sinai Medical Center, 8730 Alden Dr, W-103, Los Angeles, CA 90048, USA \\ ${ }^{2}$ Department of Psychiatry, Columbia University, New York State Psychiatric Institute, 1051 Riverside Drive, New York, \\ NY 10032, USA
}

\begin{abstract}
Post Traumatic Stress Disorder (PTSD) is highly prevalent among individuals addicted to opioids and complicates the course of illness and treatment response. Evidence-based treatments for patients with co-occurring opioid abuse and PTSD are lacking. Furthermore, little is known about the potential benefits of partial agonist therapy for these co-occurring conditions. We report the case of a patient with PTSD and opioid dependence, who experienced substantial symptomatic improvement with buprenorphine.

We conclude that screening for PTSD in the opioid-abusing population is imperative, that buprenorphine may have important therapeutic benefits, and that future studies should examine the efficacy of partial-agonist opioids for concurrent PTSD and opioid dependence.
\end{abstract}

Keywords: Post traumatic stress disorder, opioid dependence, buprenorphine, heroin, stress.

\section{INTRODUCTION}

Post Traumatic Stress Disorder (PTSD) is highly prevalent among individuals addicted to opioids. Compared to their counterparts with opioid dependence alone, patients with opioid dependence and PTSD have worse occupational functioning, physical health, and disease course [1]. Among patients going through methadone maintenance treatment, it has been estimated that over two thirds have experienced serious trauma [2], and approximately one third carry lifetime histories of PTSD [1]. Effective pharmacotherapies exist for opioid dependence and PTSD, separately, but evidence-based treatments for patients with co-occurring opioid dependence and PTSD are lacking. Furthermore, little is known about the potential benefits of partial agonist therapy. While there have been some considerations of antidepressant effects of buprenorphine [3], we are not aware of any studies or reports characterizing the effects of buprenorphine in PTSD. Here, we report the case of a patient with co-occurring PTSD and opioid dependence, who achieved remission from both diseases with buprenorphine maintenance.

\section{CASE}

$\mathrm{G}$ is a 40-year-old Puerto Rican-American man who presented to our research clinic for participation in nontreatment opioid research. $\mathrm{He}$ started using heroin intranasally at age 21 and rapidly escalated to using up to 10 bags intravenously each day. His history was notable for seeing a psychiatrist for "temper" problems as a child, and a family history of a brother addicted to heroin. He did not

*Address correspondence to this author at the Department of Psychiatry, Cedars-Sinai Medical Center, 8730 Alden Dr, W-103, Los Angeles, CA 90048, USA; Tel: 310-423-8198; E-mail: Itai.danovitch@cshs.org have a lifetime history of abusing alcohol, nicotine, or any other illicit substance. Mr. G was diagnosed with opioid dependence and admitted for participation in an opioid administration study approved by the New York State Psychiatric Institute's Institutional Review Board. The study required that Mr. G should be inducted on to buprenorphine and maintained on a dose of $8 \mathrm{mg}$ for the study duration.

G described buprenorphine as "a miracle drug". He felt euthymic, energetic, and had minimal urges to use heroin. He stated that compared to methadone, which made him feel "tired, sluggish, and a little fuzzy," buprenorphine left him feeling lucid.

On day $6 \mathrm{G}$ experienced a panic attack during a study procedure, in which he was stationed in a small room (reminiscent of a prison cell) for repeated blood draws. On careful exploration, it became clear that this episode triggered memories of a traumatic assault during a prison riot when he sustained critical injuries, extensive blood loss, and was nearly killed. At the time of the trauma he was 20 , and he went on to develop flashbacks, nightmares, and perpetual hypervigilance. $\mathrm{G}$ further clarified that his subsequent heroin use had been compelled by its ability to dampen these symptoms.

$G$ was found to meet Diagnostic Statistical Manual (DSM-IV-tr) criteria for PTSD, a diagnosis that had eluded the Structured Clinical Interview for DSMIV Axis I disorders completed at study entry, and which actually excluded him from the study [4]. Given his positive response to buprenorphine, he requested a referral to an addiction psychiatrist. He transitioned to outpatient buprenorphine maintenance with excellent results. Specifically, he reported that his sleep returned to normal, he had no flashbacks, no longer felt "jumpy," and felt free of anxiety. Whereas, in the prior two decades, his longest period of abstinence had been 
an 18-month interval of forced sobriety in prison, he now reported that his urge to use heroin was substantially diminished and was completely "clean." Greater than 20 months after his initial presentation, he was employed full time and continued to have consistent negative urine toxicology screens. He has the current diagnoses of PTSD in remission, and opioid dependence in sustained full remission on (partial) agonist therapy.

\section{DISCUSSION}

Our impression of $\mathrm{G}$ was that he had used heroin in part to self-medicate symptoms related to his underlying, previously undiagnosed, PTSD. The self-medication hypothesis suggests that drug abuse is motivated by the effort to relieve intrapsychic suffering and that there is psychopharmacological specificity to drug choice [5]. G specifically noted that heroin attenuated hypervigilance and hyperarousal, and that neither alcohol nor other illicit drugs had this effect. Furthermore, G reported that his PTSD symptoms persisted beyond the acute, subacute, and chronic withdrawal windows. Thus, his symptoms represented more than a mere artifact of a withdrawal syndrome.

G's report is consistent with reports from the literature recounting alleviation of stress-related symptoms by opioids. Studies of combat veterans suggest that use of substances often parallels the ebbs and flows of PTSD symptoms [6] Reports of non-combatant opioid addicts have also shown greater drug use severity to be associated with PTSD [7]. Among subsamples with lifetime history of PTSD, it appears that active PTSD symptoms are positively correlated with greater current drug use [2]. Finally, studies of acute opioid administration to trauma victims have suggested that shortterm administration of opioids may have some protective effect against PTSD [8].

If such reports of therapeutic benefits are compelling, why have opioids not found a formal role in the management of PTSD? The adverse consequences of chronic opioid use are considerable. In addition to the multitude of adverse effects caused by opioid dependence (detailed elsewhere), abuse of full agonist opioids causes derangements in the stress and hypothalamic-pituitary-adrenal (HPA) axis, which may exacerbate PTSD.

The HPA axis is a convergence point in the pathophysiology of both PTSD and opioid dependence. Endogenous opioids are produced in the hypothalamus -through differential slicing of proopiomelanocortin (POMC), the precursor to adrenocorticotropic hormone (ACTH) -- and help to regulate ACTH production through negative feedback inhibition [9]. When exogenous opioids are ingested, they directly suppress ACTH and glucocorticoid release [10]. This activity may help to explain the observation that opioids dampen acute stress.

However, endogenous opioids also inhibit locus ceruleus (LC) activity, counterbalancing the activating effects of corticotropin releasing factor (CRF), and attenuating the LC mediated nor-adrenergic output that is generated during a stress response. Valentino and Bockstaele speculate that because endogenous opioids are necessary for countering the stimulatory effects of CRF at the LC, opioid dependence, which can generate tolerance to opioids at the LC [11], may contribute to unopposed CRF activity at the LC, and thus, generation of a "feed-forward" stress enhancing circuit [12]. Opioid withdrawal, which itself causes increased noradrenergic outflow from the LC, greatly exacerbates this cycle, and further compounds the hyperarousal symptoms of PTSD [13]. In other words, opioid use may, on the one hand, suppresses the stress response by inhibiting HPA axis output, while on the other hand causing an imbalance of influence on the LC, favoring CRF-induced activation. Taken together with evidence that stress alters sensitivity to opioids [14], these mechanisms offer a pathway by which opioid dependence and PTSD might be mutually exacerbating.

What then might be the role for buprenorphine? Buprenorphine is a synthetic opioid with partial mu-opioid receptor agonism and mild kappa-opioid receptor antagonism. The effects of buprenorphine on the HPA axis and on PTSD are unknown, though animal data suggest that buprenorphine has far less effect on the HPA axis than full agonist opioids [15] and may facilitate HPA normalization. Therefore, buprenorphine may exert some of the therapeutic benefits of opioids without the chronic derangement that exacerbates PTSD. The kappa-antagonism of buprenorphine may also modulate the distressful affects that perpetuate PTSD psychopathology. Additionally, the putative antidepressant effects of buprenorphine are not well understood, but it is possible that they too contribute to efficacy in PTSD.

Considering the prevalence of trauma in the opioidabusing population, screening for PTSD is imperative. The paucity of efficacious treatments for this population may contribute to complacency about screening due to a sense of futility. But if our experience with $G$ has relevance for wider practice, medications, such as buprenorphine, may be found to have important therapeutic benefits. Future studies should examine the impact of buprenorphine on stress-related symptoms for patients that suffer from concurrent PTSD and opioid dependence.

\section{CONFLICT OF INTEREST}

Itai Danovitch, M.D.: NONE.

Sandra Comer, Ph.D.: Consulting arrangements with Grunethal, Johnson and Johnson, Schering-Plough, and King Pharmaceuticals.

Maria Sullivan, M.D., Ph.D.: NONE.

\section{ABBREVIATIONS}

$\begin{array}{lll}\mathrm{ACTH}= & \text { Adrenocorticotropic Hormone } \\ \mathrm{CRF}= & \text { Corticotropin Releasing Factor } \\ \mathrm{HPA}= & \text { Hypothalamus-Pituitary-Adrenal } \\ \mathrm{LC}= & \text { Locus Ceruleus } \\ \mathrm{POMC}= & \text { Proopiomelanocortin } \\ \mathrm{PTSD}= & \text { Post Traumatic Stress Disorder } \\ \text { REFERENCES } & \end{array}$

[1] Villagomez RE, Meyer TJ, Lin MM, Brown LS, Jr. Post-traumatic stress disorder among inner city methadone maintenance patients. J Subst Abuse Treat 1995; 12(4): 253-7.

[2] Clark HW, Masson CL, Delucchi KL, Hall SM, Sees KL. Violent traumatic events and drug abuse severity. J Subst Abuse Treat 2001; 20(2): 121-7. 
[3] Nunes EV, Sullivan MA, Levin FR. Treatment of depression in patients with opiate dependence. Biol Psychiatry 2004; 56(10): 793-802.

[4] American Psychiatric Association. (DSM-IV-TR) Diagnostic and statistical manual of mental disorders, $4^{\text {th }}$ ed. Text revision. Washington, DC: American Psychiatric Press, Inc. 2000.

[5] Khantzian EJ. The self-medication hypothesis of addictive disorders: focus on heroin and cocaine dependence. Am J Psychiatry 1985; 142(11): 1259-64.

[6] Bremner JD, Southwick SM, Darnell A, Charney DS. Chronic PTSD in Vietnam combat veterans: course of illness and substance abuse. Am J Psychiatry 1996; 153(3): 369-75.

[7] Hien DA, Nunes E, Levin FR, Fraser D. Posttraumatic stress disorder and short-term outcome in early methadone treatment. J Subst Abuse Treat 2000; 19(1): 31-7.

[8] Saxe G, Stoddard F, Courtney D, et al. Relationship between acute morphine and the course of PTSD in children with burns. J Am Acad Child Adolesc Psychiatry 2001; 40(8): 915-21.

[9] Howlett TA, Rees LH. Endogenous opioid peptides and hypothalamo-pituitary function. Annu Rev Physiol 1986; 48: 52736.
[10] Zis AP, Haskett RF, Albala AA, Carroll BJ. Morphine inhibits cortisol and stimulates prolactin secretion in man. Psychoneuroendocrinology 1984; 9(4): 423-7.

[11] Rasmussen K, Beitner-Johnson DB, Krystal JH, Aghajanian GK, Nestler EJ. Opiate withdrawal and the rat locus coeruleus: behavioral, electrophysiological, and biochemical correlates. J Neurosci 1990; 10(7): 2308-17.

[12] Valentino RJ, Van Bockstaele E. Opposing regulation of the locus coeruleus by corticotropin-releasing factor and opioids. Potential for reciprocal interactions between stress and opioid sensitivity. Psychopharmacology (Berl) 2001; 158(4): 331-42.

[13] Kreek MJ, Koob GF. Drug dependence: stress and dysregulation of brain reward pathways. Drug Alcohol Depend 1998; 51(1-2): 2347.

[14] Abercrombie ED, Jacobs BL. Systemic naloxone administration potentiates locus coeruleus noradrenergic neuronal activity under stressful but not non-stressful conditions. Brain Res 1988; 441(12): $362-6$.

[15] D'Elia M, Patenaude J, Hamelin C, Garrel DR, Bernier J. No detrimental effect from chronic exposure to buprenorphine on corticosteroid-binding globulin and corticosensitive immune parameters. Clin Immunol 2003; 109(2): 179-87.

(C) Danovitch et al.; Licensee Bentham Open.

This is an open access article licensed under the terms of the Creative Commons Attribution Non-Commercial License (http://creativecommons.org/licenses/bync/3.0/) which permits unrestricted, non-commercial use, distribution and reproduction in any medium, provided the work is properly cited. 